\title{
Microcomputer analyses of performance on a portable, simple visual RT task during sustained operations
}

\author{
DAVID F. DINGES and JOHN W. POWELL \\ The Institute of Pennsylvania Hospital and University of Pennsylvania, Philadelphia, Pennsylvania
}

\begin{abstract}
There is a need for brief, portable performance measures that are free of practice effects but that reliably show the impact of sleep loss on performance during sustained work. Reaction time (RT) tasks hold considerable promise in meeting this need, if the extensive number of responses they typically yield can be processed in ways that quickly provide the essential analyses. While testing the utility of a portable visual RT task during a sustained, quasi-continuous work schedule of $54 \mathrm{~h}$, we developed a microcomputer software system that inputs, edits, transforms, analyzes, and reduces the data from the RT portable audiotapes, for each 10-min trial on the task. With relatively minor modifications, the software system can be used on a minimally configured microcomputer system that supports BASIC. The software is flexible and capable of retrieving distorted data, and it generates a variety of dependent variables reflecting intratrial optimum response capacity, lapsing, and response slowing.
\end{abstract}

Some of the more useful measures of performance during sustained operations are those known to be sensitive to sleep loss; in particular, tasks that are portable and brief and that are without practice effects are most important. Reaction time tasks have the potential of meeting these three criteria. Lisper and Kjellberg (1972) demonstrated that a simple, 10-min auditory reaction time (ART) task was sensitive to 24-h sleep loss. Based on this study, Wilkinson and Houghton (1982) developed a portable, 10min simple visual reaction time (VRT) device and showed that performance on it was sensitive to as little as one night without sleep (Glenville, Broughton, Wing, \& Wilkinson, 1978).

Application of this portable VRT task to an experimental protocol involving quasi-continuous performance demands for sustained periods beyond $24 \mathrm{~h}$ has been underway in our laboratory. We are interested in determining whether aspects of VRT performance can serve as sensitive indices of sleep tendency and overall performance capacity during sustained operations. Accordingly, we sought to have VRT task performance yield in each trial dependent variables that reflected the extent of lapsing (Williams, Lubin, \& Goodnow, 1959), optimum response slowing (Dinges, Orne, \& Orne, 1983), and habituation

This research was supported in part by Office of Naval Research Contract N00014-80-C-0380 to the first author and in part by a grant from the Institute for Experimental Psychiatry. We thank Robert Wilkinson of the MRC Applied Psychology Unit, Psychophysiology Section, for developing the VRT device and for suggestions regarding its use, and Stephen R. Fairbrother for contributing to the development of the analyses; we acknowledge the helpful comments of Emily Carota Orne, Wayne G. Whitehouse, Martin T. Orne, and David A. Soskis. Reprint requests should be sent to D. F. Dinges, Unit for Experimental Psychiatry, 111 N. 49th Street, Philadelphia, PA 19139-2798.
(Kjellberg, 1977). For practicality we sought to generate these performance parameters by computer analyses of the analog VRT data.

To achieve these goals, we developed programs that allow for real time, off-line, electronic input of the VRT data, and for execution of a series of analyses designed to yield the most extensive intratrial evaluation of task performance. The programs are modularly arranged and can be used on a minimally-configured microcomputer. This flexible system allows for analysis of either transformed or untransformed data, automatic and manual editing of data, and retrieval of data distorted by low battery voltage in the VRT device. The purpose of this paper is to describe the functional characteristics of these datahandling and analysis programs.

\section{METHOD}

\section{Wilkinson Simple VRT Device}

The simple VRT test apparatus (Type RT3) was purchased commercially from K. E. Developments, Ltd., Cambridge, England, who also provided instructions and a circuit diagram. The device is a modified audiocassette recorder in which data tapes store both the RTs as inaudible $1-\mathrm{kHz}$ tone bursts and the oral annotation of the trial. Both the length of the tone burst and, more importantly, the number of $1-\mathrm{kHz}$ voltage peaks within the tone are measures of the RT in milliseconds. The stimulus is the onset of a 4-digit millisecond clock, visually displayed (3-mm LED) in a window near a built-in response microswitch. Pressing the microswitch stops the tone burst on the audiotape and the digital clock display at the RT, permitting the subject $1.5 \mathrm{sec}$ to read the value. The interstimulus interval on the task varies randomly from 1 to $10 \mathrm{sec}$. The apparatus is battery powered by $6 \mathrm{~V}$. 
More extensive information on using the device and a FORTRAN computer program for analyzing responses were obtained from Wilkinson (see Wilkinson \& Houghton, 1982). The programs reported here were developed to yield analyses other than those provided by the FORTRAN program. In addition, we sought a set of programs written in BASIC, which is currently supported by most microcomputers.

\section{Computer Hardware Required}

The programs were written on a BBC Acorn microcomputer. ' To run the programs, the minimal hardware configuration includes $32 \mathrm{~K}$ RAM, a dual disk drive, a blackand-white monitor, a printer, a parallel port, and access to two $1-\mathrm{MHz}$ internal counter/timer registers. This latter item requires that the computer have the 6522 Versatile Interface Adaptor (VIA) chip, or some equivalent chip (e.g., Z80 Counter-Timer chip) that can be controlled through an assembly language subroutine.

\section{Program Modules}

Figure 1 displays the functional characteristics of the program modules.

Data transcription. To read the data from cassette tape into the computer, an audiocassette recorder output is connected to the computer parallel port "data available flag" line which is line CB1 for the 6522 chip. This input requires TTL square pulses rather than a sinusoidal audio waveform (the standard output from a cassette recorder). The square pulse can be achieved by outputting from the earphone jack of the K. E. VRT cassette device, or from any good quality cassette recorder, with the addition of a simple pulse-squarer circuit (e.g., TTL Schmitt Trigger) between the recorder output and the computer. The recorder should have a variable electronic motor speed control, which is a common feature.

An assembly language subroutine that controls the 6522 VIA parallel port chip assumes that the input pulse has a 1 -msec period and measures RT by counting the pulses until a gap of $3 \mathrm{msec}$ is detected, whereupon the program returns to BASIC with the accumulated count in two bytes of storage. A second assembly language subroutine is used with data that has been frequencydistorted due to low battery voltage in the portable device during data collection. ${ }^{2}$ This subroutine measures the du- ration of the tone envelope rather than the number of discrete pulses comprising the tone. ${ }^{3}$

The BASIC program repeatedly calls the selected subroutine for RTs until three RTs of less than $100 \mathrm{msec}$ are encountered, whereupon the program for data input is automatically terminated. Occasional RTs of $100 \mathrm{msec}$ or less exceed the human performance limit, but may occur as a result of a premature (false start) response coinciding with stimulus onset. The program displays RTs of $100 \mathrm{msec}$ or less, but does not store them as part of the data trial. Three consecutive RT values equal to or below $100 \mathrm{msec}$ virtually always signal the playback of speech annotation audio (preceding the next trial). ${ }^{4} \mathrm{By}$ timing the duration of a trial in this manner, rather than by simply accumulating $10 \mathrm{~min}$, varying trial lengths can be accommodated.

Although the data transcription programs do not use cumulative time in trial to execute trial termination, cumulative time for each response is collected (using the TIME function in BASIC) for the purpose of minute-by-minute analyses (see below). In addition, the programs flag the first and last RTs in a trial by storing them as negative values. The negative value serves to inform the experimenter and the subsequent analysis programs that these RTs will not be included. The reason for excluding the first and last RTs in a trial is that these values are often contaminated by handling the portable RT device at the beginning and end of the trial.

Data editing. Use of the programs on data collected during sustained operations revealed that the automatic negative flags set for certain VRTs did not provide all of the data editing needed. Occasionally manual editing is required (e.g., when the audio tape runs out before a trial is ended; when a trial length exceeds the arbitrary trial length limit; or when the second VRT in a trial is delayed by machine handling). For these reasons, the system permits the user to manually edit data following transcription. Data can be added to or subtracted from a trial file, or the data for an entire trial can be keyed into the computer, assuming the data are available in another reliable form.

Data transformation. Once editing is complete, the operator has the option of continuing analyses of the raw data or of data that are reciprocally (one/RT) transformed. The reciprocal transformation option is included at this

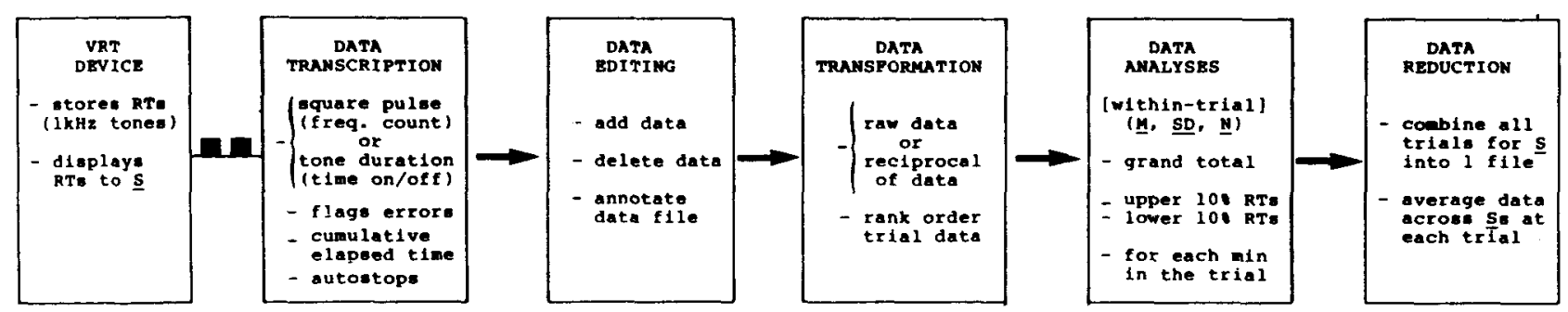

Figure 1. Functional sequence in which program modules process visual reaction time performance data obtained from a portable VRT device. 
point to deal with the problem of proportionality between the square of the mean and the standard deviation of RT data in a trial (see Kirk, 1968, pp. 63-67). (Other transformations can be substituted with only a very minor modification of the program.) Following this, data from the VRT trial (e.g., 10-min trial) are rank-ordered from smallest to largest value within the trial. The user has the option of printing the rank-ordered data at this time. An option is being added that permits saving these rankordered data for other special analyses, such as nonparametric statistics.

Data analyses. Following transformation, the data in a trial file are subjected to a number of analyses. The grand trial mean, variance, and standard deviation are calculated. The same variables for the upper $10 \%$ and lower $10 \%$ VRTs in the trial are generated, to yield data on the increase in lapses as well as on changes in the optimum response capability within the trial. The system then generates the mean, variance, standard deviation, and number of responses for each consecutive minute in the trial by using the cumulative time data obtained at transcription. This minute-by-minute analysis yields data relevant to the rate of response slowing or habituation within each trial. The results of these analyses are then saved as a new trial data file, which can also be printed.

Data reduction. At this stage, the user may combine the data trials for a given subject into one summary data file, along with additional information, such as the number of false starts in each trial and the time of day each trial occurred for that subject. For example, if a subject performed 17 10-min VRT trials during a period of $54 \mathrm{~h}$ of sustained wakefulness with quasi-continuous work demands, then the final trial data file for that subject would contain the summary results for all 17 trials.

After combining results across trials (within a subject) into one file, the user then has the option of combining data across subjects into a group file. Executing this option results in the mean, variance, and standard deviation being calculated across subjects, using each subject's mean value, for the grand mean of a trial, the mean for the upper and lower $10 \%$ of responses in a trial, and the mean for each consecutive minute in the trial. The final file summarizes average performance on each variable for each trial for a group of subjects. It is, of course, crucial that the user specify the appropriate trial file numbers for group combination. This requires that all of the subjects being averaged into a group have the same number of trials, and that these trials occurred at approximately the same time of day or same circadian phase. Deviations from this requirement are handled by appropriate labeling of trial files for individual subjects before this stage of data processing.

\section{DISCUSSION}

The impact of involuntary sleep onsets on performance during sustained operations is one of the key issues surrounding the use of work schedules that extend beyond the 24-h day. Much research has focused on the assessment of sleep tendency during sustained operations through the development of tasks that are sensitive to involuntary microsleeps. The most sensitive of these tasks, however, are not necessarily the most practical to use in actual field situations. Rather, simple portable RT devices, such as those devised by Wilkinson and Houghton (1982), hold considerable promise for providing an index of sleep tendency, if the data are analyzed to yield the maximum information. Automation of appropriate analyses further enhances the practicality of these devices.

The analyses and programs reported here were developed to use with a simple VRT device during a sustained work schedule. The device and the microcomputer analysis system were extensively tested in an experimental protocol involving quasi-continuous work for $54 \mathrm{~h}$. An option was included that allows for the recovery of data distorted by low battery voltages in the VRT device, a problem often encountered when portable devices are used.

The data analyses contained in the programs involve traditional measures of both response lapsing (i.e., slowest $10 \%$ of VRTs in a trial) and response slowing (i.e., fastest $10 \%$ ). Beyond this, however, the programs offer data suitable for nonparametric statistical analyses, and more importantly, they yield a measure of response change or habituation (cf. Kjellberg, 1977) within each trial (i.e., minute-by-minute analyses). We have recently extended analyses in this latter regard by developing programs that fit least squares linear regression lines to the response change data within a trial.

The results of the various analyses generated by this system are now being compared within and between 40 subjects who were repeatedly tested during $54 \mathrm{~h}$ of quasi-continuous work under conditions of sustained wakefulness. In addition, the VRT data will be compared to changes in self-reported mood, in body temperature, and in other performance tasks recorded during the period. The results from these comparisons should indicate the extent to which the VRT task variables incorporated in the programs yield a sensitive, valid, and reliable measure of sleep tendency during sustained operations. The ultimate goal of this approach is to develop a performance measure of sleep tendency that can be used for predicting the ability of persons to function effectively at different times of the day throughout a sustained work period.

\section{REFERENCES}

Dinges, D. F., Orne, M. T., \& Orne, E. C. (1983, March). Does the lapse hypothesis account for all sleep deprivation effects? Paper presented at the 54th annual meeting of the Eastern Psychological Association, Philadelphia, PA.

Glenville, M., Broughton, R., Wing, A. M., \& Wilkinson, R. $T$. (1978). Effects of sleep deprivation on short duration performance measures compared to the Wilkinson Auditory Vigilance Task. Sleep, $1,169-176$.

KJELlberg, A. (1977). Sleep deprivation and some aspects of performance: II. Lapses and other attentional effects. Waking \& Sleeping, 1, 145-148. 
KIRK, R. E. (1968). Experimental design: Procedures for the behavioral sciences. Belmont, CA: Wadsworth.

Lisper, H., \& KJELLEe RG, A. (1972). Effects of a 24-hour sleep deprivation on rate of decrement in a 10-minute auditory reaction time task. Journal of Experimental Psychology, 96, 287-290.

Wilkinson, R. T., \& Houghton, D. (1982). Field test of arousal: A portable reaction timer with data storage. Human Factors, 24, 487-493.

Williams, H. L., Lubin, A., \& Goodnow, J. J. (1959). Impaired performance with acute sleep loss. Psychological Monographs, 73(14, Whole No. 484).

\section{NOTES}

1. The BBC Acorn computer was used because it had the most powerful BASIC and the most flexible I/O access of the microcomputers available in our laboratory. The progamming was done by J. W. Powell. More specific information about the analyses and copies of the programs are available by writing to the authors.

2. This distortion is clearly audible to the user during playback of the tape and indicates the need to call in the alternative measurement subroutine. The K. E. Developments, Ltd. VRT device is powered by four 1.5-V C-size alkaline batteries. The device we received had no battery voltage indicator. Although the accompanying instructions state that batteries should be changed when the 4-digit display disappears, we observed that, when the supply voltage drops below $4.4 \mathrm{~V}$, the display is still clear, but the crystal-controlled 1-kHz RT clock generator begins to slow. Thus, the frequency of the VRT tone slows, resulting in an underestimation (by frequency count) of the RT times. The VRT clock (tone) continues to oscillate more slowly, down to $3.3 \mathrm{~V}$, whereupon the display disappears. In repeated data collection, frequency distortions of up to $30 \%$ were noted. The cassette device's tape transport mechanism, on the other hand, remained quite stable throughout these voltage drops, and slowed only marginally below $3.4 \mathrm{~V}$. This meant that frequency-distorted RT data was still available on the tapes in the form of overall duration of the tone envelope. Hence the alternate subroutine for duration measurement was developed. The duration measure has remarkably little distortion due to motor speed variations during recording and playback. This was validated by comparing the duration and frequency VRT measures when the cassette voltage was above $4.5 \mathrm{~V}$ In such cases, the millisecond agreement was very high; for example, of 146 consecutive VRT times, the two measures never disagreed by more than $2 \mathrm{msec}$, and were identical or within $1 \mathrm{msec} 98.6 \%$ of the time. Although the frequency measure is preferable when battery voltage is high, the duration measure serves to salvage distorted data. One final caveat should be mentioned. If a recorder other than the $\mathrm{K}$. E. VRT device is used to read the data into the computer, and the duration subroutine is required, it is essential that the tape transport speed of this second recorder be adjusted to match that of the VRT device to insure accurate duration measurements.

3. This measurement is accomplished by using one of the VIA timer registers as an accurate $1-\mathrm{msec}$ clock. The routine continues to count this internal reference clock while external pulses are being received, regardless of the momentary period of the pulses. Again, a 3-msec gap between pulses signals the end of the pulse train, and the routine returns to BASIC.

4. While coincident false starts are recorded on the audiotape as extremly short RTs, false starts that do not coincide with stimulus onsets do not get recorded on the tape at all. Although false starts are relatively rare in a 10-min trial on the task, they do occur. The only way that an accurate count of the number of false starts can be obtained is by requiring the subject to say "false start" aloud when one occurs. The subject knows this has happened because the stimulus display shows a zero value. To gather both false start data and the number of times subjects made reading errors from the display, we routinely have them state all response times aloud during performance trials. 